\title{
Proposed Framework for Determining Added Mass of Orion Drogue Parachutes
}

\author{
Usbaldo Fraire Jr., CPAS Analysis Engineer $^{1}$ \\ Jacobs Technology, Houston, TX, 77598 \\ James Dearman, Parachute Systems Analyst ${ }^{2}$ \\ Lockheed Martin, Houston, TX, 77058 \\ Aaron Morris, CPAS Analysis IPT Chair ${ }^{3}$ \\ Barrios Technology, Houston, TX, 77598
}

\begin{abstract}
The Crew Exploration Vehicle (CEV) Parachute Assembly System (CPAS) project is executing a program to qualify a parachute system for a next generation human spacecraft. Part of the qualification process involves predicting parachute riser tension during system descent with flight simulations. Human rating the CPAS hardware requires a high degree of confidence in the simulation models used to predict parachute loads. However, uncertainty exists in the heritage added mass models used for loads predictions due to a lack of supporting documentation and data. Even though CPAS anchors flight simulation loads predictions to flight tests, extrapolation of these models outside the test regime carries the risk of producing non-bounding loads. A set of equations based on empirically derived functions of skirt radius is recommended as the simplest and most viable method to test and derive an enhanced added mass model for an inflating parachute. This will increase confidence in the capability to predict parachute loads. The selected equations are based on those published in "A Simplified Dynamic Model of Parachute Inflation" by Dean Wolf. An Ames 80x120 wind tunnel test campaign is recommended to acquire the reefing line tension and canopy photogrammetric data needed to quantify the terms in the Wolf equations and reduce uncertainties in parachute loads predictions. Once the campaign is completed, the Wolf equations can be used to predict loads in a typical CPAS Drogue Flight test. Comprehensive descriptions of added mass test techniques from the Apollo Era to the current CPAS project are included for reference.
\end{abstract}

\section{Nomenclature}

CPAS = CEV Parachute Assembly System

$\mathrm{C}_{\mathrm{d}} \quad=$ drag coefficient

$\mathrm{CEV}=$ Crew Exploration Vehicle

$\mathrm{D}_{\text {norm }}=$ normalized diameter; $\operatorname{sqrt}\left(\mathrm{C}_{\mathrm{x}} \mathrm{S} / \mathrm{C}_{\mathrm{x}} \mathrm{S}_{\mathrm{o}}\right)$

$\mathrm{D}_{\mathrm{o}} \quad=$ nominal diameter

$\mathrm{D}_{\mathrm{p}} \quad=$ projected diameter

Gen II $=$ Second Generation

$\mathrm{K}=$ added mass coefficient

$\mathrm{m} \quad=$ mass

$\mathrm{m}_{\mathrm{A}} \quad=$ added mass

$\mathrm{q}_{\mathrm{bar}}=$ Dynamic Pressure

$\mathrm{R}_{\mathrm{p}} \quad=$ projected radius

${ }^{1}$ CPAS Analysis Engineer, Aerothermal and Flight Mechanics, 455 E. Medical Center Blvd., Webster, TX., Future AIAA Member

${ }^{2}$ Parachute Systems Analyst, Landing and Recovery Systems, 901 Bay Area Blvd, Houston, TX, AIAA Member

${ }^{3}$ CPAS Analysis IPT Chair, Aerothermal and Flight Mechanics, 455 E. Medical Center Blvd., Webster, TX, AIAA Senior Member 


$\begin{array}{ll}\mathrm{v} & =\text { velocity } \\ \mathrm{V} & =\text { Volume } \\ \mathrm{W} & =\text { weight } \\ \rho & =\text { density } \\ \theta & =\text { flight path angle }\end{array}$

\section{Introduction}

A

full understanding of the aerodynamics of parachute inflation has eluded parachute engineers for decades. One particularly vexing topic is that of added mass. In 1991, Cockrell wrote that the "dearth of test data" made a detailed understanding of added mass virtually impossible and concluded that the "[added] mass concept has little more to offer to contemporary parachute design." Twenty years later the authors of this paper are in a similar state of frustration. There simply are not enough data to quantify relevant added mass terms.

Flight simulations are currently used to predict parachute riser loads for both the Orion vehicle and parachute structural design. In addition, these flight simulations will be used to ultimately verify the CPAS flight performance requirements. ${ }^{2}$ The CPAS project must verify load requirements with simulations ${ }^{2}$ because it is challenging to test

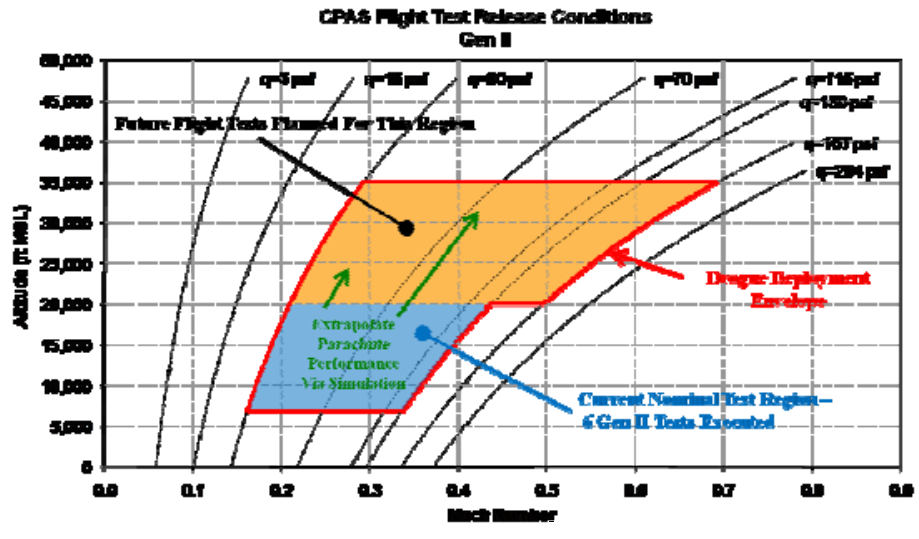

Figure 1. CPAS Flight Test Release Conditions throughout the entire deployment envelope. ${ }^{3,4}$ Figure 2 shows the current CPAS Drogue deploy envelope. A total of six Gen II flight tests have been executed under the $20,000 \mathrm{ft}$ region shaded in blue and include single and cluster parachute configurations. ${ }^{5}$ Due in large part to limited confidence in the derivation of the heritage added mass models, there is risk associated with extrapolating performance derived at lower altitudes and true airspeeds to the orange shaded region. Therefore, a test campaign is planned in the deployment envelope above $20,000 \mathrm{ft}$ to help reduce this risk by empirically determining performance in this regime. To satisfy these testing needs, CPAS is currently developing state-of-the-art balloon test techniques and a new Parachute Compartment Drop Test Vehicle (PCDTV) for the Engineering Development Unit (Gen III) tests. The following three items will help reduce the risk posed by extrapolating current performance: 1) complete high altitude test campaign, 2) reduce the required drogue deploy altitude, and 3) increase the confidence in the added mass model. Option 3, increasing the confidence in the added mass model, is the focus of this paper.

Testing for the Apollo Program was markedly different from the CPAS project. The Apollo Program completed extensive testing throughout its nominal envelope, represented by the blue shaded region in Figure 1. The success of this program, even with simple loads prediction methods, was in part a tribute to the thoroughness of their test program. Even as early as 1945, Von Karman recognized that the added mass should have a substantial impact on load predictions as the altitude is increased. ${ }^{6}$ This is significant because CPAS must currently use flight simulations to extrapolate parachute performance parameters to untested

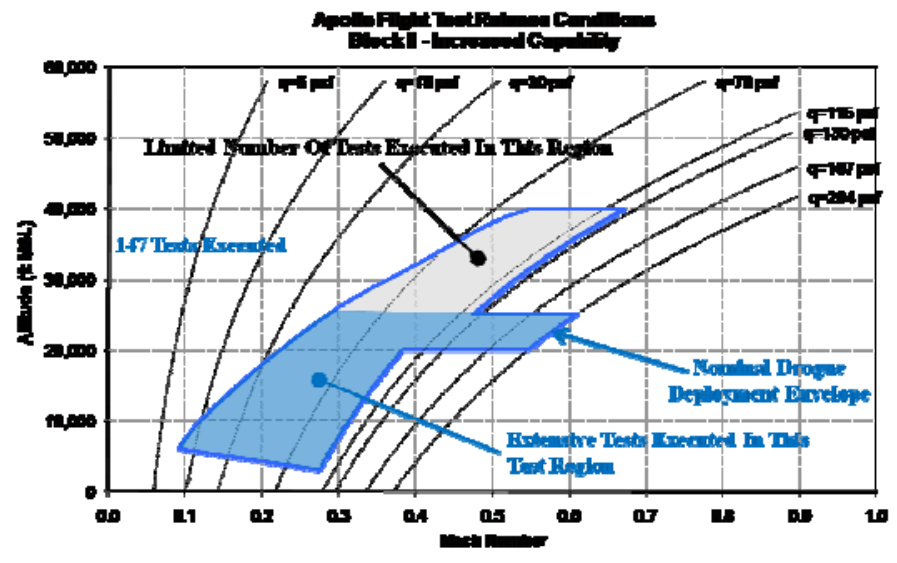

Figure 2. Apollo Flight Test Release Conditions 
altitudes and true airspeeds. Even with limited testing in the upper right regime, we must have confidence that the simulations can bound expected performance. As a result, CPAS desires well understood and testable added mass terms for delivering bounding load predictions.

Multiple organizations are involved in the design of Orion. As a result, load predictions are produced by different flight simulations. The two primary flight simulations are $\operatorname{DSS}^{7}$ and DCLDYN. ${ }^{8}$ Each has a different heritage and uses different added mass models. Unfortunately, load predictions are highly sensitive to these differences. Both are anchored to past programs and flight test results; however, the differences result in distinct parachute inflation parameters for the same tests. The CPAS project decided to enforce a common added mass model in the two primary simulations. Neither model was particularly advantageous, but due in part to its superior documentation, the CPAS project chose to emulate the DCLCYN model in the DSS simulation. This emulator is a temporary solution. The differences in loads predictions left the CPAS project with a desire to test and quantify contributions of added mass, and eventually develop a more physics-based model.

A deeper understanding of added mass will help increase confidence that the simulation loads predictions ultimately used to verify system requirements are bounding and accurate. A High Altitude Balloon (HAB) test remains a priority as it will expand the understanding of parachute performance at the higher altitude and higher true airspeed flight conditions. HAB test data can even be used with heritage versions of the added mass model to improve confidence in predicted loads, similar to Apollo. However, due to the limited number of tests planned, parachute analysts must also understand the range of expected loads. This understanding can be acquired by leveraging test data from the lower altitude / lower mach regime. This extrapolation technique carries less risk with improvements to added mass.

Any future program that requires parachute system operation in conditions above $25,000 \mathrm{ft}$ will face similar decisions between expensive testing and trying to improve the confidence in simulations. Improvements in the understanding of added mass will have an impact beyond CPAS. This paper intends to initiate a discussion within the broader community.

Ultimately, the goal of this paper is to strongly echo Cockrell's 1991 conclusions. There is a "dearth of test data," and the authors recommend conducting the wind tunnel test campaign outlined in Section III. The gathered data will ultimately help to verify a set of equations from Wolf ${ }^{9}$ and Macha ${ }^{10}$. Once these equations are anchored via wind tunnel tests, then the predictive capability can be tested in flight.

\section{Equations of Motion}

For symmetrical bodies, there are fully 21 non-negligible components of added mass. ${ }^{1}$ Because the purpose of this discussion is the improvement of predicted parachute inflation loads, the scope is limited to the added mass that exerts its influence along the flight path. Even normal to the flight path, Macha ${ }^{10}$ confirms that the added mass terms may be neglected because "the resulting small error in system orientation within the gravitational field negligibly affects the large forces and accelerations of interest during the inflation process."

The literature often uses inconsistent nomenclature when describing the various nuances of added mass. For example, the enclosed and included mass are used interchangeably, but refer to the same air mass within the canopy as illustrated in the appendix Section F. In this paper, added mass refers to the total additional inertia observed in an accelerating parachute. The added mass of a parachute is often decomposed into enclosed and apparent air masses. These added mass components account for the inertia of the air within the canopy and that resulting from interactions with the air in the immediate vicinity, respectively.

For a parachute system in ballistic descent, $\mathrm{Knacke}^{11}$ and many others have derived the following relationship for parachute force by application of the classical expansion of Newton's second law for a system with variable mass:

$$
F=1 / 2\left(C_{x} S\right)_{p} \rho v^{2}+\left(m_{A}+m_{v}+m_{p}\right) \frac{d v}{d t}+v \frac{d m}{d t}+W_{V} \sin \theta
$$

Thus, the added mass will exert two distinct influences on a parachute system. First, the inertia of the system will be larger than the sum of the payload and parachute masses. Second, if the added mass is changing, there will be an additional impulse due to the exchange of mass across the system boundaries. Simply, the effects of added mass are relegated to periods of system acceleration or mass change. Unfortunately for the design engineer, it turns out that the very events that drive the strength and weight of parachute elements - disreefing and inflation events (even so called 'infinite mass' inflations) - intrinsically define such periods. Therefore, any integrated parachute simulation that attempts to capture parachute loads and predict system performance during these events over a range of conditions is required to address added mass. Moreover, any proposed added mass formulation must specifically 
address both the quantity of mass at a given state, and the evolution of added mass over these critical periods. (It may come as a surprise to some that in current practice the time derivative of the added mass and the added mass rate provided to the system equations of motion are not always one and the same.)

Given the somewhat sensitive and controversial nature of added mass, several useful approximations have developed over time, driven by theory, geometry, and empirical observation. The heritage approaches of Apollo and the UD233 simulation are discussed, as well as the DCLDYN and DSS algorithms currently used by CPAS. Finally, an appeal is made for a common approach first proposed by Wolf ${ }^{9}$ that better presents itself for the prediction of loads and the derivation of appropriate input parameters.

\section{A. Apollo Heritage}

During the development of the Block I and Block II configurations, Apollo parachute loads were calculated alternately by use of opening shock factors and dynamic drag area growth curves dependent on canopy type and reef stage. ${ }^{12}$ Though the methods did not specifically address added mass, the effects were included by definition, as they were derived for each test condition independently. Coupled with an extensive test program that covered the operational deployment envelope of the Apollo parachutes, these methods resulted in a highly successful parachute system.

However, in a subsequent investigation ${ }^{12}$, it was recognized that the inclusion of added mass was desirable to improve the accuracy of predicted inflation loads and two models were developed in tandem. The first, the Mass/Time method, predicts the total parachute added mass as a function of the drag area at a given instant in time:

$$
m_{A}=K_{A} \rho\left(C_{x} S\right)^{3 / 2}
$$

Three simple assumptions are made. The first is that the added mass can be determined from knowledge of the canopy shape and radius.

$$
m_{A}=K \rho V
$$

This assumption is well established and common to most treatments of added mass. Second, the ratio, $\mathrm{K}_{\mathrm{A}}$, is treated as a constant; because it requires a constant shape throughout inflation, this assumption limits applicability of the method to the latter stages of inflation. Finally, the canopy projected radius is assumed proportional to the square root of the drag area. Though reasonable at first glance, this assumption couples the added mass rate to the drag area rate. Thus, any discontinuous change in drag area rate (as was present in the linear fill equations used by Apollo) has the effect of producing large and unreasonable forces. Thus, the method was not satisfactorily developed for the full range of reefed stages and parachutes. (The coupling problem is not unique to Apollo and is revisited in Part D of this section.)

The second approach, the Shape/Distance method overcomes this complication by uncoupling the added mass from the drag area. First described in detail by Rust ${ }^{13}$, the theory does not require the projected radius as an input, but derives added mass as a function of distance along the flight path by considering the net flux of air into and out of the canopy for various stages of inflation. Alternatively, this data can also be acquired from film analysis of a test (and this is the approach that the Apollo investigation takes.) ${ }^{12}$ The parachute volume for the added mass calculation in Equation 3 may then be calculated by the selection of an idealized parachute shape. Rust suggests the use of a conical frustum and either a prolate or oblate ellipsoid. The value of $\mathrm{K}$ may be derived from test data or a desired potential flow solution as a function of the projected radius, $R_{p}$. Notably, Wolf ${ }^{9}$ and Macha ${ }^{10}$ have criticized Shape/Distance methods for disregarding the criteria and assumptions required to ensure dynamic similarity.

\section{B. UD233/DSS}

The current incarnation of the DSS simulation, presently maintained by NASA, traces its heritage to a 6-DOF dynamic model developed in the 1960's and used to simulate entry and descent during the Viking program. ${ }^{14} \mathrm{~A}$ more modern variant called UD233A was used extensively during the Shuttle SRB recovery project. The added mass implementation of this simulation remains largely unchanged in DSS, although some significant disagreement exists between the included source code and documentation. ${ }^{15}$

Using Heinrich's added mass model ${ }^{16}$, the UD233 User's Guide divides the added mass into apparent and enclosed masses. Both quantities are proportional to a volume defined by the cube of the parachute projected radius. The enclosed mass takes the parachute volume as a hemisphere:

$$
M_{e}=\frac{2 \pi}{3} \rho R_{p}^{3} .
$$

This is a case, unfortunately, where the documentation and the source code disagree. The code adds what appears to be a cylindrical term of unknown derivation that vanishes as the parachute reaches full inflation. 


$$
M_{e}=\rho\left[K_{p} \frac{2 \pi}{3} R_{p}^{3}+\frac{1}{2 \pi} D_{p}^{2} D_{o}\left(1-D_{\text {norm }}\right)\right]
$$

Such additional volume terms are widespread in the literature ${ }^{21}$ but this specific cylindrical formulation has not manifested itself in the current review. To obfuscate matters further, DSS adds a ratio to the given enclosed mass that scales the final value in Equation (5) by $90 \%$.

As in Equation (3), the apparent mass is given as a ratio of the enclosed canopy volume:

$$
M_{a}=K_{p} \pi \rho R_{p}^{3},
$$

where $K_{p}$ is assumed equal to a constant 0.25 in the documentation. The paper cites Heinrich and Noreen ${ }^{16}$ as a reference for this value, with no mention of the fact that for an inflating parachute, it should be a function of normalized time and not constant due to parachute shape change. The source code places the value of the same constant at 1.25, which appears somewhat suspicious given that the enclosed mass is already accounted for. It is notable as well, that, as in Equation (2), UD233 and DSS assume that the projected radius is proportional to the square root of the drag area (see section D).

This code in particular serves as a prime example of how a heritage tool with gaps in documentation and references can leave future users in a state of bewilderment. The situation is made more disorienting by the knowledge of the resoundingly successful parachute systems the tools have enabled. The discussion begs the following questions:

1. How much success was due to the predictive accuracy of the simulations and how much was due to thorough test programs and extensive reconstruction/validation efforts?

2. Do empirical data or theories exist that might substantiate the aforementioned inconsistencies observed in the code and the accompanying manual?

3. How applicable are the changes to a range of parachute canopy types and reefing ratios? What assumptions were made in their derivation that might invalidate simulation results for specific cases?

\section{DCLDYN}

DCLDYN $^{8}$ is a proprietary tool developed and owned by Airborne Systems. With roots in the latter years of the Apollo program, iterations of this tool have been used during the development of several successful parachute systems, including the Space Shuttle Orbiter drag chute, a variety of drone recovery systems, Kistler K-1 spacecraft recovery system, Space X Dragon Capsule ${ }^{18}$, and the Pad Abort Demonstrator programs. ${ }^{19}$

The DCLDYN added mass model also distinguishes between enclosed and apparent masses but treats the enclosed mass as the summation of a conical frustum and a hemisphere defined by $\mathrm{R}_{\mathrm{p}}$. According to an Apollo investigation $^{12}$, this shape traces its heritage back to a Heinrich paper which has not been found in the present search. ${ }^{20,21}$ The source for $\mathrm{R}_{\mathrm{p}}$ is not derived in the documentation. The apparent mass is derived from potential flow solutions of ellipsoids of equivalent volume. This procedure likely traces its heritage to a Neustadt ${ }^{22}$ paper which has also eluded discovery. As $\mathrm{R}_{\mathrm{p}}$ increases, the approximating ellipsoid evolves from a prolate to an oblate configuration in order to fulfill the volume and $\mathrm{R}_{\mathrm{p}}$ constraints. It is notable that both Wolf ${ }^{9}$ and Macha ${ }^{10}$ have criticized potential flow solutions for their inapplicability to real fluids.

\section{Added Mass Rate}

In both $\mathrm{DSS}^{7}$ and DCLDYN ${ }^{8}$, the added mass rate is filtered so that sharp changes in the time derivative of $\mathrm{R}_{\mathrm{p}}$ do not produce equivalent sharp changes in mass rate. These filters are either proprietary or not well documented and it is unclear how the underlying physics was validated. For the case of DSS, the projected parachute radius has been coupled to the drag area. Such coupling requires a smooth transition at the boundaries of parachute inflation such that a discontinuous rate of change of $\mathrm{R}_{\mathrm{p}}$ does not translate to unrealistic loads. By itself, this problem would suggest that CPAS is improperly using the simple drag area growth curves by expecting them to translate well to parachute volume growth. Instead of relying on filters to uncouple the predicted added mass rate from the rate provided to the equations of motion, a simpler solution would be a model that predicts smooth behavior from the outset and does not rely on a presupposed relationship between canopy volume and drag.

\section{E. A Simplified Dynamic Model}

In 1973, Wolf 9 proposed an inflation model that uniquely included a radial momentum conservation equation for the parachute skirt. This addition allowed the prediction of skirt area, not by an assumed air mass flux or a relationship to drag area growth, but instead based solely on data resolvable from steady state wind tunnel data. In 1993, Macha ${ }^{10}$ refined the derivation and reduction of input parameters. An investigation of the procedure yields the 
following required input parameters for the model: $\mathrm{C}_{\mathrm{x}} \mathrm{S}$, the nominal drag area tangent to the flight path, $\mathrm{C}_{\mathrm{r}} \mathrm{S}$, the drag term that resists the canopy inflation, and the instantaneous canopy volume all as a function of skirt radius, $\mathrm{R}$. Two empirical added mass functions must also be derived from experiments: $K_{r}$ and $K_{x}$, such that the added mass in the radial and tangential momentum equations are given by:

$$
\begin{aligned}
& m_{A r}=K_{r} \rho V \\
& m_{A x}=K_{x} \rho V .
\end{aligned}
$$

The authors believe that, due to its more fundamental empirical approach and reduced reliance on assumed shapes and potential flow theory, Wolf's approach presents the most promise for a unified inflation/added mass framework with "true predictive capability."

There are a number of empirical parameters associated with this model. In lieu of the mostly undocumented assumptions and derivations present in current simulations, it is desirable to experimentally determine the applicable parameters to proceed with load prediction efforts. The test techniques required to determine these parameters are described presently.

\section{Measurement Test Techniques}

Various heritage test techniques were evaluated to determine a viable test series to quantify added mass. A summary of the heritage test techniques that were researched and not selected for this recommendation can be found in the appendix. Ultimately, the simplified dynamic model proposed and refined by Wolf ${ }^{9}$ and Macha ${ }^{10}$, respectively, was selected by the authors as the best method for determining the added mass contributions during parachute inflation events. Macha's proposal for deriving the added mass coefficients found in Equations (7) and (8) is a more fundamental approach than the other researched techniques. In fact, it appears possible to correlate the added mass parameters with previous drop test data given the volume, $\mathrm{C}_{\mathrm{x}} \mathrm{S}$, and $\mathrm{C}_{\mathrm{r}} \mathrm{S}$ as a function of the skirt radius, R. Moreover, it appears to deftly overcome a central problem with the Heinrich and Apollo tests: the assumption that the apparent mass coefficient, $\mathrm{K}$, in a non-inflating configuration is unchanged when applied to an inflating parachute.

Macha used seven intermediate reefed stages in a wind tunnel test to acquire correlations for $\mathrm{C}_{\mathrm{x}} \mathrm{S}$ and $\mathrm{C}_{\mathrm{r}} \mathrm{S}$ as a function of $\mathrm{R} . \mathrm{C}_{\mathrm{r}} \mathrm{S}$ was derived from the reefing line tension and suspension line angle acquired for each intermediate stage. The instantaneous canopy volume term as a function of skirt radius was derived using scaled parachute data. Today, an improved canopy volume function may be acquired with photogrammetry used during full-scale wind tunnel testing. The proposed test architecture is shown in Figure 3. Note that the figure is not intended to limit the test configuration to seven stages; because CPAS Drogues are reefed parachutes, more than seven intermediate stages would likely be required. The wind tunnel test configuration shown is for a single Orion Drogue test article. A reefing line is used at the skirt of the canopy to control the volume during the test. Tensiometers are rigged into the riser and reefing line. The camera is used to determine the canopy volume as a function of skirt radius. Once the $\mathrm{C}_{\mathrm{x}} \mathrm{S}, \mathrm{C}_{\mathrm{r}} \mathrm{S}$, and volume functions are known, the derivation of the added mass functions $\mathrm{K}_{\mathrm{x}}$ and $\mathrm{K}_{\mathrm{r}}$ is executed by comparing flight test data with a simulation model with appropriate initial conditions. The new proposed procedure explicitly gives the drag area, $\mathrm{C}_{\mathrm{x}} \mathrm{S}$, (and thus the drag force) as a function of the skirt radius, R. Finally, the residual added mass component of force is used to iteratively determine the correct functions for $\mathrm{K}_{\mathrm{x}}$ and $\mathrm{K}_{\mathrm{r}}$. 

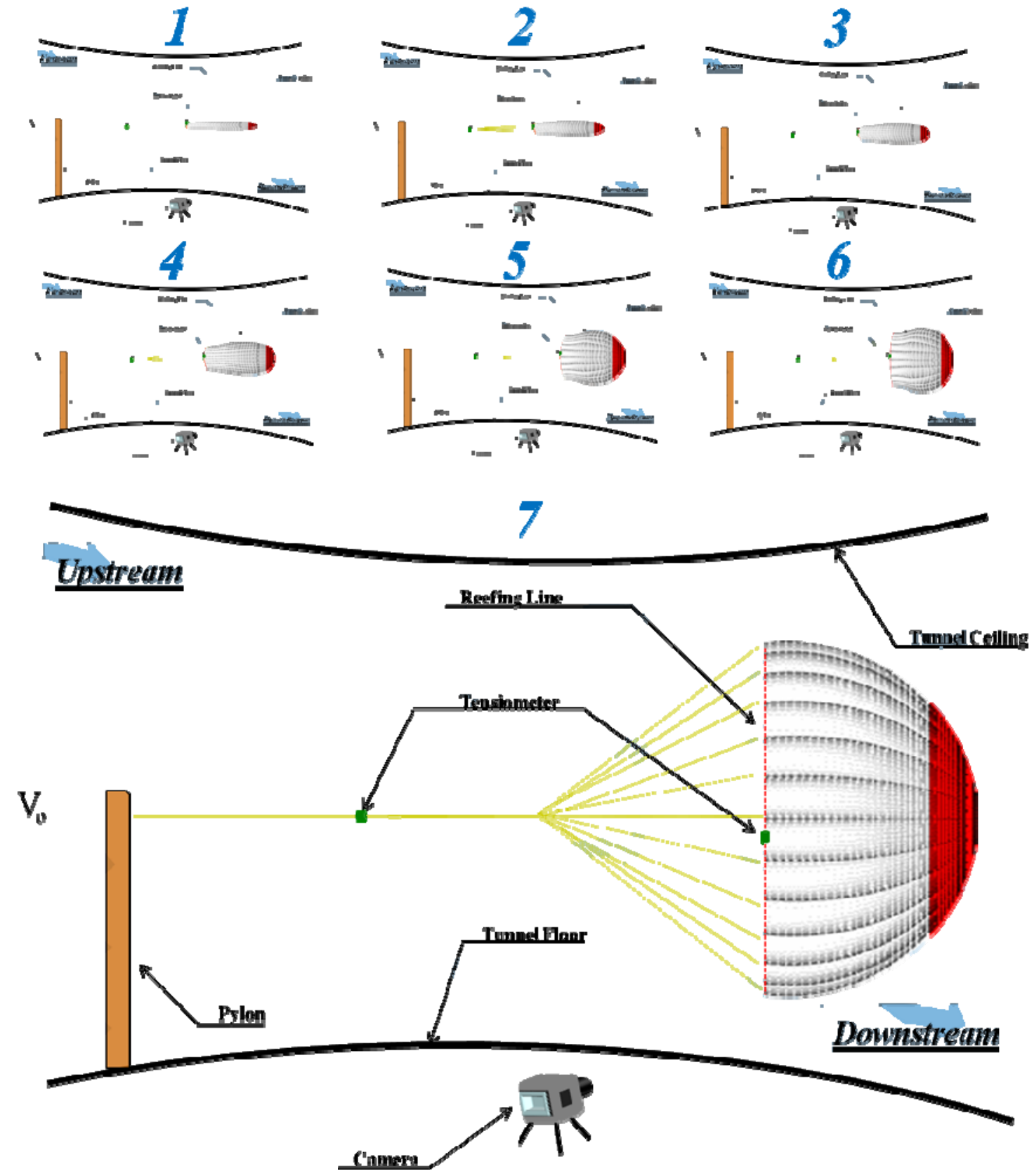

Figure 3.Recommended Wind Tunnel Test Technique

Executing the proposed test technique does involve limitations. If the Ames 80x120 wind tunnel is used to provide the testing environment the Mach number and $\mathrm{q}_{\mathrm{bar}}$ will be relatively low compared to the realistic deployment environment. If a higher speed wind tunnel test is desired, a smaller-scale Drogue or Main will be required. Scaling parachutes contribute another level of complexity and introduces new problems associated with implementing a scaling technique. A Main parachute could be tested in the Ames 80x120 wind tunnel for the smaller reefed inflation phases only. The larger inflation events near the full-open phase will require flight testing to obtain an understanding of the added mass effects. 


\section{Conclusion}

Parachute designers have identified a lack of data related to parachute added mass. This paper has recommended an Ames 80x120 test campaign be conducted with a CPAS Drogue to quantify terms in an enhanced added mass model based on the Wolf equations. Executing higher altitude tests, reducing the drogue deploy altitude, and completing the recommended wind tunnel tests will reduce uncertainty in loads predictions and reduce the risk posed by extrapolating current flight simulations. This strategy will ultimately increase confidence in the robustness of the CPAS hardware.

\section{Acknowledgments}

The authors wish to acknowledge Dr. Jean Potvin of St. Louis University for his assistance in the understanding of added mass equations and literature. The authors also wish to thank Chuck Lowry, retired Apollo Earth Landing Systems parachute program manager at North American Aviation (Rockwell), for his assistance in the literature search.

\section{Appendix}

Developing a measurement test technique to quantify the effects of added mass during parachute inflation events is a complex and involved problem. Various test and measurement concepts have been proposed to provide a viable test solution for quantifying this parameter by space programs and parachute design engineers. The measurement test techniques proposed for ground and flight tests have consisted of three recurring fundamental test design elements. These include measuring a deceleration, riser tension force, and using a fixed canopy shape. Each heritage added mass test technique included some combination of the three fundamental test design elements. A comprehensive list is included to document the evolution of proposed added mass test techniques from the Apollo Era to the current CPAS project. The comprehensive list of the proposed test techniques are included as reference material only and serve as additional documentation to provide the reader with an insight to the research results attained during the development of this paper.

\section{A. Two Payload Weight Method (Von Karman and Heinrich)}

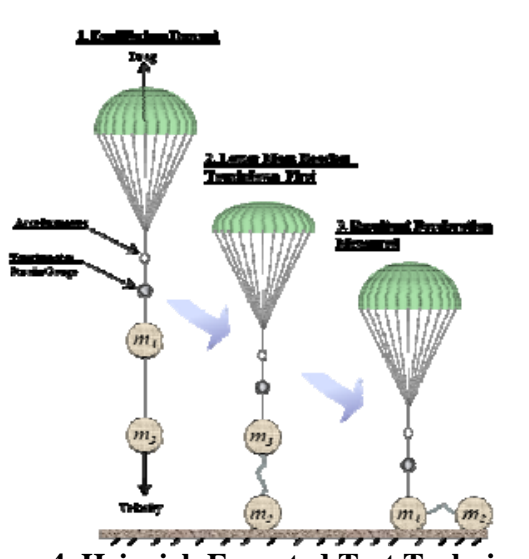

Figure 4. Heinrich Executed Test Technique

Von Karman suggested that the apparent mass of parachutes can be quantified by releasing half the suspended weight of the system and measuring the resulting deceleration. ${ }^{12}$ The model test used to execute von Karman's proposed flight test concept was comprised of deploying various small scale parachutes using two separate payload weights connected by riser lines. The design of the test configuration ensured the lower payload weight reached touchdown first while the system was in equilibrium descent. ${ }^{12}$ The response of the system to a sudden mass and load change involved an experienced reduced gravity force and resulting deceleration on $m_{1}$ and the parachute as shown in Figure 4. The propulsion the canopy experiences when the lower mass reaches touchdown is explained as a transfer of kinetic from the surrounding medium, air, to the object, parachute. ${ }^{17}$ Conversely, the transfer of kinetic energy from an object to a medium results in an experienced deceleration. The amount of additional air mass was measured by the degree of the deceleration in combination with the released, $m_{2}$, and remaining load, $m_{1}$. The instrumentation used to measure the deceleration and tension force in the riser lines during these events were an accelerometer and strain gauge, respectively. The strain gauge and accelerometer sensors were attached to the riser line that connects to $m_{1}$. The test technique included two of the three design elements mentioned. This technique did not require a parachute with a fixed canopy shape and was deployed straight to full open. A series of tests were executed with different types of parachutes that included solid flat, extended skirt, personnel guide surface, stabilization guide surface, and ribbon configurations. ${ }^{17}$ 


\section{B. Apollo Program Methods}

The Apollo program emphasized the importance of understanding the effects of added mass on parachute loads and recommended several test techniques for future test teams to use as a building block to quantify this parameter. Added mass of a parachute canopy could not be inferred from typical flight data and requires special techniques to acquire measurements in a wind tunnel or flight test. Assumptions made included assuming the added mass and drag area are unique functions of the state of the parachute opening. Equally, the state of the parachute opening is a unique function of the distance the parachute has traveled since the beginning of inflation. ${ }^{12}$ More importantly, added mass is variable during inflation and can only be quantified for a single instant in the inflation process. The following recommended wind tunnel and flight tests were intended to quantify the variables $A_{1}$ and $A_{2}$ of Equation 9. In incompressible, irrotational, potential flow, Wolf $^{12}$ derives the following relationship for the added mass along the flight path: where $\mathrm{s}$ is the distance along the flight path. The coefficients $\mathrm{A}_{1}$ and $\mathrm{A}_{2}$ are both functions of the projected radius.

$$
m_{A}=A_{1}+A_{2} \frac{d R_{p}}{d s}
$$

As mentioned, $A_{1}$ is dependent of canopy shape and is associated with acceleration of the fluid by the canopy relative to the system CG due to canopy shape changes. $A_{2}$ is dependent on both canopy shape and the rate of change of canopy shape and is associated with acceleration of the fluid by the canopy relative to the system CG due to canopy shape changes. ${ }^{12}$ A separate technique was proposed to measure the $\mathrm{A}_{2}$ term and is also discussed.

\section{1) Apollo Wind Tunnel Test for $A_{1}$}

The wind tunnel test technique used to measure the added mass term, $A_{1}$, included all three observed

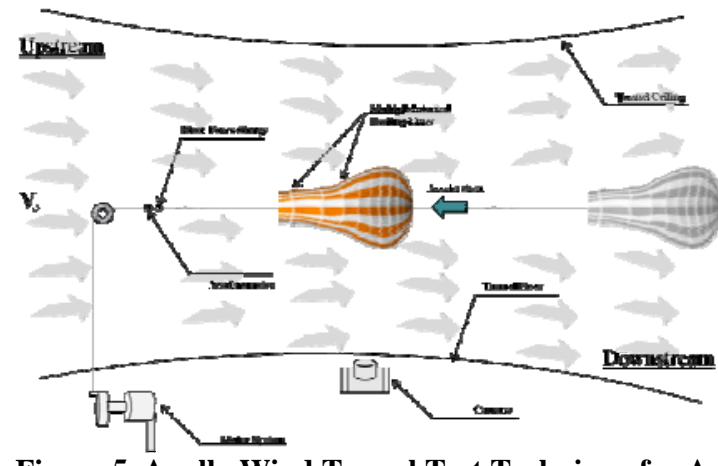
fundamental test design elements which are using a fixed canopy shape and measuring a deceleration plus riser tension force. A small scale parachute such as a Drogue is meant to be used for this test technique. The fixed canopy shape is sustained by including internal reefing lines to characterize an instant in the inflation process. A motorized pulley system is used to accelerate the fixed shape canopy upstream in a wind tunnel environment with a constant free stream velocity, $\mathrm{v}_{\mathrm{o}}$. The speed of the motor system is altered until the canopy oscillates foreand-aft at a high enough velocity to acquire a deceleration and riser force measurement. ${ }^{12}$ The velocity

Figure 5. Apollo Wind Tunnel Test Technique for $A_{1}$ of the oscillation is measured by integrating the accelerometer data. ${ }^{12}$ The varied velocities must be significant enough to allow a riser force to vary from its mean value by at least \pm 10 percent. The remaining riser line of the system is collected at an eccentric arm on a flywheel that is located outside of the wind tunnel. The instrumentation used on the test configuration includes a riser force gauge and accelerometer installed in series along the riser line as shown in Figure 5 A motion camera is used to record the inflation phenomenon as the canopy re-inflates after the sudden deceleration and obtain position data used for verifying the accelerometer output through differentiation. The instrumentation and camera system are time synched for comparing captured camera inflation events and acquired measurements. No test vehicle is required for this configuration. The important factors of the wind tunnel test technique are canopy shape, which correspond to different instants in opening, canopy type, forebody shape, and free stream velocity. Free stream velocity is significant because changes would result in a variation in the streamline field in and around the canopy. ${ }^{12}$ This test technique is limited by the high dynamic pressure conditions that are unattainable in a wind tunnel to simulate an actual deployment conditions.

\section{2) Apollo Wind Tunnel Test for $A_{2}$}

A second wind tunnel test technique was proposed for quantifying the second term, $A_{2}$, associated with the rate of change of the canopy shape in Equation 9. The $A_{2}$ term is estimated to be approximately one-half of the $A_{1}$ term and the contribution of $A_{2}$ to the added mass Equation 9 is relatively small and can be estimated in terms of $A_{1}$ with reasonable accuracy. ${ }^{12}$ Performing a test to measure the $\mathrm{A}_{2}$ term is not practical, but instructive to understanding the added mass contributions during the inflation process. The proposed test technique for measuring $\mathrm{A}_{2}$ includes a fixed canopy shape, riser tension force, and camera system. This technique does not require an acceleration measurement because it is focused on understanding the rate of change of the canopy mouth. The proposed canopy 


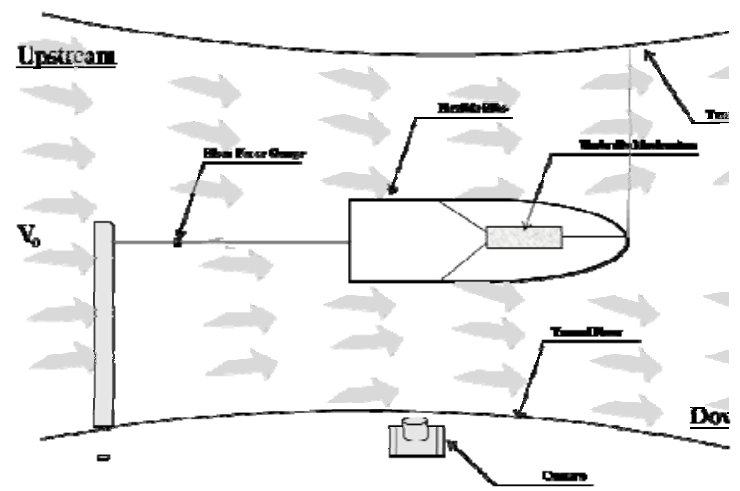

Figure 6. Apollo Wind Tunnel Test Technique for $\mathbf{A}_{2}$

construction includes flexible ribs along each radial of the parachute as shown in Figure 6. The second element of the canopy requires an umbrella mechanism that is comprised of an air cylinder used to generate a simulated oscillation experienced during the inflation process. The air cylinder has three spokes connected to the crown and radials of the flexible canopy shape. A transverse support wire connected at the tunnel ceiling is used to support the canopy mass. The riser force gauge is installed just below the confluence point on the riser line. Data acquired from the riser force gauge and camera system are used to interpret and quantify $\mathrm{A}_{2}$ performance. This test technique provides a postulation that a method for measuring the added mass terms for an inflating parachute, $A_{1}$ or $A_{2}$, is achievable, and must be evaluated separately to understand the relationship described in Equation 9.

\section{3) Apollo Flight Test for $A_{1}$}

The test techniques investigated by the Apollo program included a flight test for acquiring measurements for the $\mathrm{A}_{1}$ added mass term described in Equation $9 \mathrm{~A}$ flight test was advantageous because it allowed a method for measuring the added mass of large scale parachutes, such as an Apollo Main, at the full-open stage. A wind tunnel test technique limited this measurement capability to only smaller scaled parachutes. Another wind tunnel limitation was operating at a high dynamic pressure to simulate a similar deployment environment. ${ }^{12}$ Data measurements on how parachute added mass varies at high dynamic pressures provides propitious data that can be used to quantify this parameter. This test technique included the three fundamental test design elements which are using a fixed canopy shape, and measuring a riser force plus a deceleration. The canopy construction represents an instant in the inflation process and its fixed shape was sustained with the use of internal reefing lines. A riser force gauge and accelerometer were installed just below the confluence point on the short riser as shown in Figure 7. The short riser line was then rigged to a set of two harness lengths that connect to the aft attach points of a fin stabilized bomb test vehicle. Harmonic variation in the riser force

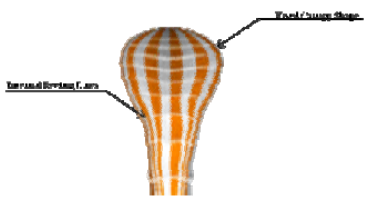
must be packaged within the vehicle. As a result, a proposed test vehicle was comprised of an interrupted falling weight mechanism located at the aft end of the vehicle to generate oscillating D'Alembert forces and create similar harmonic variations a pulley system would provide to a canopy accelerated in a wind tunnel. ${ }^{12}$ The falling weight mechanism was a device that descends through the body of the bomb at a variable velocity. ${ }^{12}$ This bomb-like vehicle provided the required deceleration to quantify added mass. The mass transfer generated by the oscillation would allow riser and deceleration measurements to be acquired. A pitot tube was included on the nose of the proposed test vehicle configuration to acquire static and stagnation pressure measurements. The success criteria for this proposed test technique was to acquired a minimum of $6-12$ oscillations to quantify added mass. The important test variables included the canopy shape/size, fore body shape, free stream dynamic pressure, and Mach number.

\section{B. Earlier Investigated CPAS Box Weight Stack Test Technique}

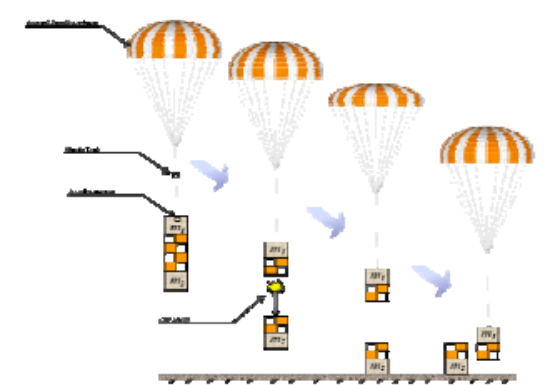

Figure 8. Box Weight Stack Test Technique

The box weight stack method can be used for quantifying the added mass of an Orion Drogue or Main parachute. The following technique will be specific to an added mass test for a $116 \mathrm{ft}$ Orion Main. Elements from Heinrich's methods described in Section A are applied by releasing half the suspended weight. The payload weight system is comprised of four stacks each weighing $2.5 \mathrm{Klbs}$ for a total payload weight of $10 \mathrm{Klbs}$. The payload weight is driven by the design limit load of a single Main, 10 Klbs. Each stack are attached using current rigging methods and include an accelerometer on-board $m_{1}$ to acquire measurements during the mass release events as shown in Figure 
8. The second mass, $\mathrm{m}_{2}$, would be released after the parachute system executes the nominal reefing schedule, reaches the full open phase, and is allowed time to reach steady state. A pyrotechnic mechanism would be required to separate the masses. At the arrival of these conditions, half of the suspended weight is released. This would allow measurements of the deceleration and riser force. The canopy could also be permanently reefed at an optimized reefing stage for measuring the added mass. A single or cluster of two Drogues could be executed using this approach.

\section{Earlier Investigated CPAS Wind Tunnel Test Technique}

A wind tunnel test campaign for measuring the added mass of an Orion parachute would look similar to the proposed Apollo method from Section B.1. The availability of a larger scale wind tunnel such as the Ames 80x120 is an advantage the Orion program has over the Apollo era which was limited to an $80 \times 40$ wind tunnel. A larger wind tunnel, such as the Ames $80 \times 120$, allows the testing of all intermediate Drogue inflation stages including the full open phase. The same capability is not available for a Main. An Orion Main would require wind tunnel analysis of the smaller reefed stages prior to full open with an additional flight test required to complete the testing campaign for the larger reefed and full open inflation stages. Fundamental test design elements used for this test technique

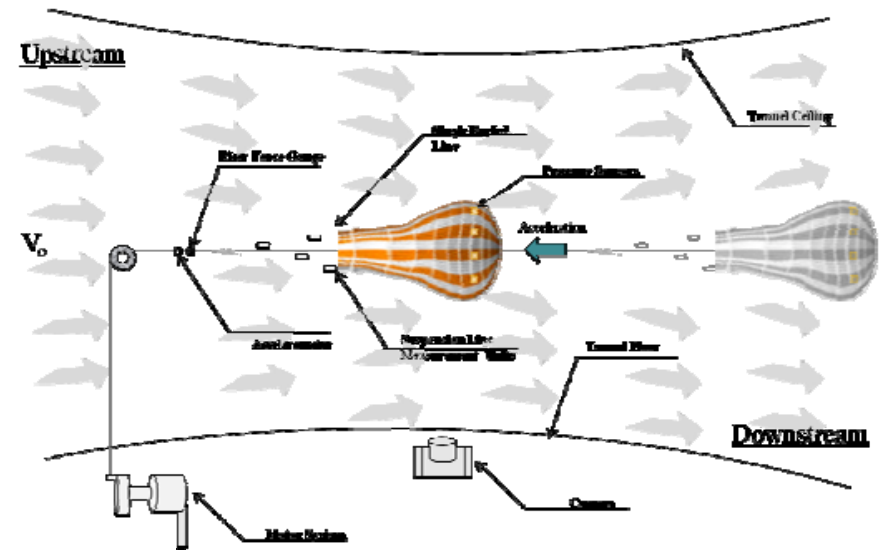

Figure 9. Investigated CPAS Wind Tunnel Test Technique include using a fixed canopy shape and measurement of a riser tension force plus an acceleration. The fixed canopy shape of an Orion parachute would not include internal reefing lines in the construction to control the volume, but instead a single reefed line to characterize an instant in the inflation process. This approach would eliminate test operation concerns for damaging the valuable parachute article. Additional information on the canopy pressure distribution would be advantagous to acquire with the use of pressure sensors on the canopy. A strain link for measuring the riser tension force is located on the riser line below the confluence point with additional suspension line measurement units above the confluence point for redundant tension force measurements. An accelerometer unit is rigged into the riser line to acquire the appropriate acceleration measurements. The measurement technique for collecting added mass data of a single Orion parachute would require a motorized pulley system to accelerate the parachute through the free stream velocity similar to the Apollo approach. As the parachute is accelerated a specified distance and allowed to rest the parachute would experience a deceleration and a measurement during the deceleration events would be acquired. The resulting deceleration could be measured for various test scenarios. A pulley system allows various speeds, wind velocities, and angle of attack capabilities to be utilized. A combination of high speed and high definition cameras are positioned near the canopy to capture the inflation events. The mentioned technique for measuring added mass effects is limited by the low dynamic pressure conditions that are attainable in a wind tunnel.

\section{Earlier Investigated CPAS Flight Test Technique 1 - MDTV/CMS}

A practical flight test technique for measuring the added mass of Orion parachutes is developed using fundamental test design elements from cited legacy sources. A two body system is a recurring test design element required in a flight test to measure a resulting deceleration when half the suspended weight is released. The proposed test vehicle to execute an Orion added mass test is comprised of a Medium Drop Test Vehicle (MDTV) on a Cradle Monorail System (CMS). An MDTV $\backslash$ CMS vehicle system is currently used to execute single Drogue and Main parachute tests and are typically extracted from a C-130A. The CMS in this proposed test technique is the vehicle that acquires the added mass data. Previous understanding of added mass techniques required half the suspended weight of a vehicle to be dynamically released to generate a significant mass and load change. For this specific technique, the MDTV will ballasted to 7,000 lbs and CMS will remain its current weight, 12,000 lbs, to resolve any test operation challenges. The design limits of a Main parachute test are not considered only a practical test technique solution. Furthermore, a better understanding of inflation parameters has led to propose a less dynamic mass release to create the deceleration required to quantify added mass. The rationale for a less intense mass change 
is meant to understand the sensitivity of the system. The MDTV and CMS vehicles are equivalent to $m_{2}$ and $m_{1}$ from

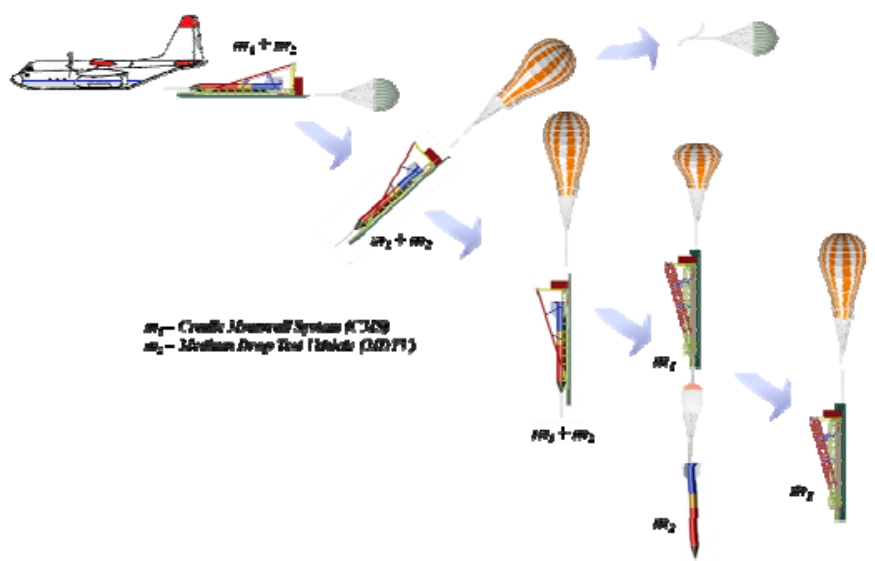

Figure 10. Investigated CPAS Flight Test Technique 1 Heinrich's test scheme in Figure 10. The release of $\mathrm{m}_{2}$ would occur at a $90^{\circ}$ angle to ensure quality measurements are acquired on the CMS. A fixed canopy shape test design element is adopted and a Main would be reefed to a specified reefing ratio to characterize a single instant in the inflation process. Internal reefing lines as mentioned in section B.2 would not be ideal for an Orion Main and result in damage to a costly article. An avionics tray on board the CMS with accelerometers, data acquisition sensors, and a $30 \mathrm{~K}$ Strain Link or TMS unit on the riser of the test parachute could be used to acquire the minimum data needed. All accelerometer sensors would be installed on the CMS rather than on the riser line as previous techniques

proposed. This test may possibly be executed each time a MDTV test was required to understand single parachute performance and allow the CMS to acquire a statistical amount of measurements. There are indicators that large ringsail parachutes behave differently than medium sized or small ringsail parachutes and therefore it is believed that information on added mass scale effects would be desirable ${ }^{\text {Error! Bookmark not defined. }}$.

\section{E. Earlier Investigated CPAS Flight Test Technique 2 - Sub-Dart System}

A secondary flight test technique for measuring added mass using the MDTV/CMS combination would require the MDTV to be modified with capabilities to release a sub-dart system at the nose of the cone section. The cone section is comprised of removable vehicle weights that are used to weight the system up or down if required. Similar to the Earlier Investigated CPAS Flight Test Technique 1, the test vehicle would be extracted from a $\mathrm{C}-130 \mathrm{~A}$ and added mass measurements would be acquired on the MDTV and CMS as described in the previous section. An Orion Drogue or Main may be used to acquired the data, but the large scale parachutes are recommended for flight testing. The MDTV would include a Drogue parachute to reach a desired test condition and static line deploy a Main parachute at a decided optimum reefed stage. At the time the MDTV reaches steady state under the Main or Drogue parachute, half of the suspended weight, or as close to half the suspended weight as operationally possible, would be released from the nose section of the

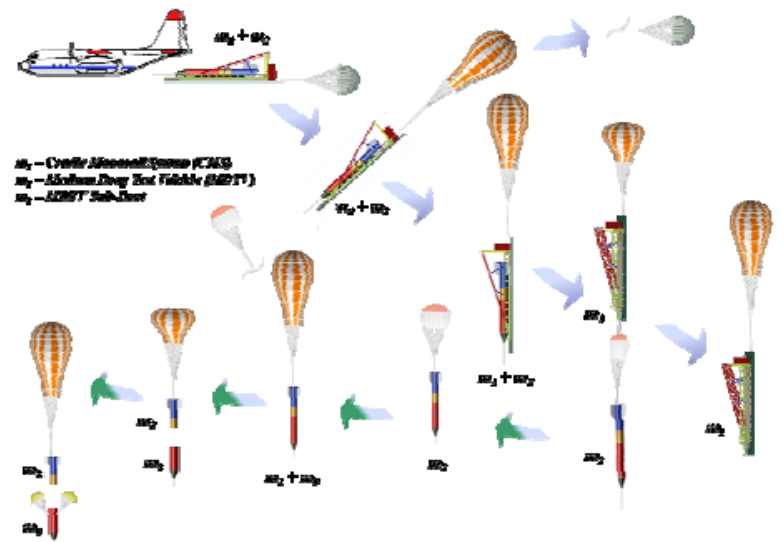

Figure 11.Investigated CPAS Flight Test Technique 2 MDTV. If no recovery system for the released sub-dart system is required the released body would simply reach touchdown and accelerate away from the remaining MDTV. If a recovery system is required for the sub-dart system, two $15 \mathrm{ft}$ drag augmentation parachutes could be deployed to allow the nose section of the vehicle to fall at a higher rate compared to the fin portion of the vehicle and deploy a Drogue parachute when their separation distance are at an acceptable distance. An avionics tray on board the fin section of the MDTV would acquire the necessary velocity, acceleration, data acquisition sensors for measuring the resulting deceleration. A strain link or TMS unit would be used to measure the riser force during these events. This specific test technique would acquire two added mass data points. Using a different canopy shape and/or payload weights may be advantageous for understanding the added mass effects on the systems. 


\section{F. Additional Conceptual Added Mass Visuals}

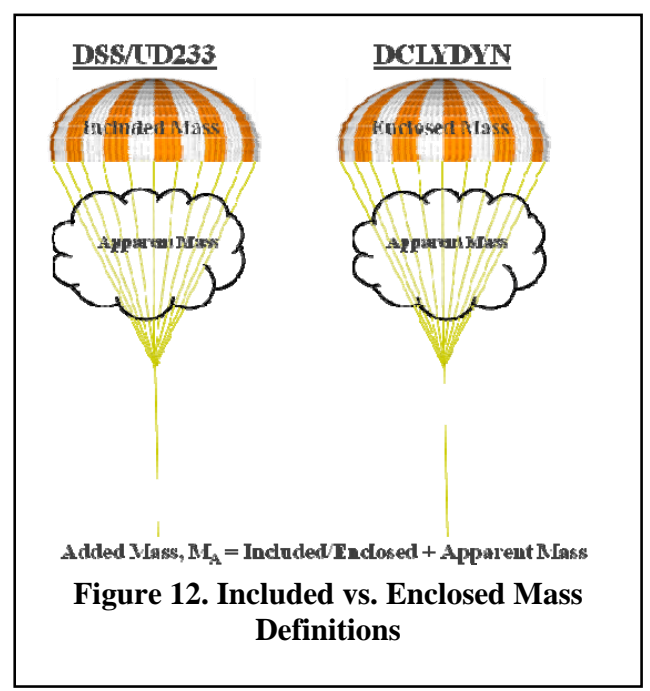

\section{References}

${ }^{1}$ Cockrell, D. J., “Apparent Mass - Its history and its Engineering Legacy for Parachute Aerodynamics,” University of Leicester, England, AIAA-91-0827-CP, 1991.

${ }^{2}$ Morris, A. and Olson, L., "Verification and Validation Plan for Flight Performance Requirements on the CEV Parachute Assembly System," 21st AIAA Aerodynamic Decelerator Systems Technology Conference and Seminar, Dublin, Ireland, May 2011 (submitted for publication).

${ }^{3}$ Ray, E., "Challenges of CPAS Flight Testing," 21st AIAA Aerodynamic Decelerator Systems Technology Conference and Seminar, Dublin, Ireland, May 2011 (submitted for publication).

${ }^{4}$ Morris, A., et al., "Development of New Drop Test Vehicles and Test Techniques for the Orion CEV Parachute Assembly System," 21st AIAA Aerodynamic Decelerator Systems Technology Conference and Seminar, Dublin, Ireland, May 2011 (submitted for publication).

${ }^{5}$ Morris, A., et al., "Summary of CPAS Gen II Parachute Performance," 21st AIAA Aerodynamic Decelerator Systems Technology Conference and Seminar, Dublin, Ireland, May 2011 (submitted for publication).

${ }^{6}$ Karman, T. V., "Note on Analysis of the Opening Shock of Parachutes at Various Altitudes," Linda Hall Library, Kansas City, MO, Call No: TL752. V66 quarto.

${ }^{7}$ Cuthbert, P. A. and Desabrais, K. J., "Validation of a Cargo Airdrop Software Simulator," AIAA Aerodynamic Decelerator Systems Technology Conference and Seminar, Monterey, California, May 2003.

${ }^{8}$ Taylor, A.P. and Murphy, E., "The DCLDYN Parachute Inflation and Trajectory Analysis Tool," Irvin Aerospace Inc., Santa Ana, CA, 92704 AIAA 2005-1624.

${ }^{9}$ Wolf, D., "A simplified Dynamic Model of Parachute Inflation," J. Aircraft, Vol. 11, No. 1, Jan. 1974, pp. 28-33, AIAA-73-450.

${ }^{10}$ Macha, J.M., "A Simple, Approximate Model of Parachute Inflation," Sandia National Laboratories, Albuquerque, NM 87185, AIAA-93-1206.

${ }^{11}$ Knacke, T.W., "Parachute Recovery Systems Design Manual,” U.S. Navy Report NWC TP-6575, Para Publishing Co., Santa Barbara, CA, 1992.

${ }^{12}$ Mickey, F.E., et al.,"Investigation of Prediction Methods for the Loads and Stresses of Apollo Type Spacecraft Parachutes," Northrop Corporation, Ventura Division Newbury Park, California, 91320, NVR-6431.

${ }^{13}$ Rust, L.W., Jr., "Theoretical Investigation of the Parachute Inflation Process," NVR-3887, July 1965, Northrop Corporation, Ventura Division, Newbury Park, CA.

${ }^{14}$ Corwin, B., "Chute Modeling Background: UD233," IPT Presentation, Analysis IPT, 18 Feb. 2010.

${ }^{15}$ Moog, R. D., et al., "Parachute Simulation User's Guide Computer Program UD233A," Martin Marietta Corp, Denver Aerospace Division Denver, CO, February 1986.

${ }^{16}$ Heinrich, H. G., and Noreen, R. A.," Analysis of Parachute Opening Dynamics with Supporting Wind Tunnel Experiments," University of Minnesota, Minneapolis, Minnesota, AIAA 68-924. 
${ }^{17}$ Heinrich, H. G., " Experimental Parameters in Parachute Opening Theory,” University of Minnesota, Minneapolis, Minnesota, 1953

${ }^{18}$ URL: http://www.spacex.com/ [cited February 23, 2011]

${ }^{19}$ URL: http://www.spacenews.com/ [cited February 23, 2011].

${ }^{20}$ Heinrich, H.G., "Some Research Efforts Related to Problems of Aerodynamic Deceleration," WADD TN-60276,Wright-Patterson Air Force Base, Ohio, Nov. 1961.

${ }^{21}$ Rust, L. W., Jr., "Performance of and Design Criteria for Deployable Aerodynamic Decelerators," Project No. 6065, Task No. 606503, Wright-Patterson Air Force Base, OH, Dec. 1963.

${ }^{22}$ Neustdat, M., Eriksen, R.E., and Guiteras, J.J., "Apollo Recovery System Dynamic Analysis," NVR-3528, April 1964, Northrop Ventura, Newbury Park, CA. (Note: This report is proprietary to the Space and Information Division of the North American Rockwell Corp.) 